\title{
Friend Relationship Weighting for Academic Performance Prediction on University Delegation at Following Competition
}

\author{
Eva Firdayanti Bisono, Tora Fahrudin, and Joko Lianto Buliali
}

\begin{abstract}
Academic performance is an important key to student success or failure. Therefore, academic performance prediction become a popular research on education. In general, several researches used GPA to predicting academic performance. However, there are some aspect that also plays a role in student academic performance, like friend relationship. So, this paper will analyze the correlation between academic performance and friend relationship. Friendship will be seen from communication frequency between students when become University delegation. Each students friend will have weight to show their closeness. In this paper, proposed method gives friendship weight based on communication frequency proportion between student among all student in one faculty. Indeed, close friends have a higher weight than other friends. So, the friendship weight sorted into descending order to get the closest friend. Then, their GPA convert into academic label, i.e. cumlaude, excellent, very good, or drop out. Furthermore, label will be compared to obtaining validation of our hypotheses that friendship plays a role in academic performance achievement. We use scholar student delegation dataset in competition from year 2015 in 7 study programme with 160 scholar students. Experimental results showed that the proposed method can predict academic performance $43 \%$ from the total data sample.
\end{abstract}

Keywords-Academic Performance, Co-occurrence, Friendship, Weighting, Friendship Network.

\section{INTRODUCTION}

A cademic performance is the important key to student success or failure. It plays an important role on developing student to become contributor or leader for his country. Furthermore, academic performance can also affect student future such as job seeking or study period [1]. Therefore, academic performance prediction become a popular research on education. In general, Grade Point Average (GPA) is used in several research for academic performance prediction. Practically, there have been some external factors which can affect academic performance prediction. So, there have been many researches used external factors such as mobile usage data, internet usage [2], or friend relationship. They combined the external factor with Grade Point Average (GPA) for academic performance prediction.

Friend relationship is one of external aspect that can be used for academic prediction. Friends are formed from a relationship between two people in spend time together, have same social value, and a mutual respect of each other. In general, a close friend has positive and negative effects for academic performance. A close friend usually gives some advice or motivation to each other while they attend academic process. Furthermore, student with high academic performance, tend to have a close friend with high academic performance too or vice versa [3].

Friend relationship can't be a constant basis for academic predictions. Because friend relationship is a dynamics social relationship. A friend relationship may change over time [4]. This can be caused by several aspects such as their interactions. These aspects can increase trust and the level of intimate [4].

Friendship modelling can be extracted from their interaction between each other at the same location. A close friend, tend to be at the same location because of their same interest [5]. This behavior activity can be the

Eva Firdayanti Bisono, Tora Fahrudin, and Joko Lianto Buliali are with Departement of Informatics, Institut Teknologi Sepuluh Nopember, Surabaya, 60111, Indonesia. E-mail: joko@cs.its.ac.id.

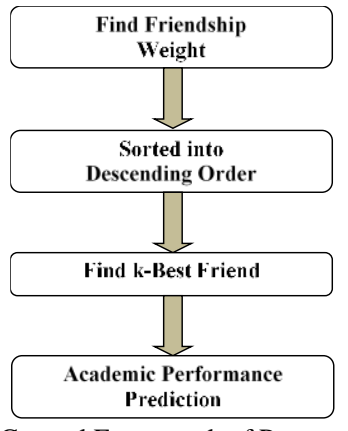

Figure 1. General Framework of Proposed Method.

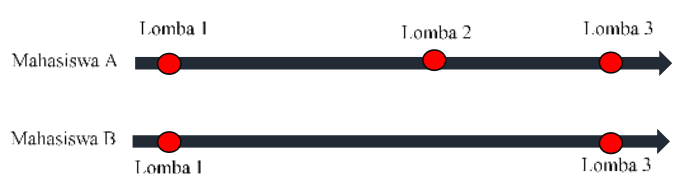

Figure 2. An Illustration of Co-occurrence Theory.

basis for extracting interaction activities between each other at the same location. Extraction process used cooccurrence. Co-occurrence is demonstrated to yield records when two students at the same location [1].

Intuitively, the increasing co-occurrence frequency indicate that two students are close friends [5]. Cooccurrence represent the number of interaction activity each student. However, the major challenge in used friendship for academic performance is the difficulties of data collecting of real friends. The number of students becoming delegation in competition is very small. It is possible that the number of meetings between students will be the same. So, we need weighting method which can calculate communication frequency ratio between students. This communication frequency become friendship weight. To get the list of each student close friend, we sort the friendship weighting in descending order. Then, friend academic performance predicate, will be the prediction result in this research.

In this paper we proposed a friend relationship weighting for academic performance prediction on university delegation at following competition based on 
TABLE 1.

EXPERIMENTAL RESULT FOR EFFECT OF THE NUMBER OF FRIENDS

\begin{tabular}{ccc}
\hline \hline Location & Number of $\mathbf{k}$ & Accuracy (\%) \\
\hline Competition & 1 & 32 \\
& 3 & 32 \\
& 5 & 32 \\
& 7 & 32 \\
\hline
\end{tabular}

\begin{tabular}{|c|c|c|c|c|c|c|c|c|}
\hline ID & M00000 & M00001 & M00002 & M00003 & M00004 & M00005 & M00006 & M00007 \\
\hline M00005 & $\# 1 ;$ & \# & $\#$ & $\#$ & $\#$ & $\#$ & $\# 1 ;$ & \# \\
\hline M00029 & $\#$ & \#6; & \# & \# & \# & \# & $\#$ & $\# 7$; \\
\hline M00047 & \# & \# & \# & \# & \# & \# & \# & \# \\
\hline M00048 & \# & \# & \# & \# & \# & \# & \# & \# \\
\hline M00050 & $\# 2 ;$ & \# & $\#$ & $\#$ & $\#$ & $\# 1 ;$ & $\#$ & $\#$ \\
\hline
\end{tabular}

Figure 2. A Few Samples of Co-occurrence Matrices Dataset.

interaction modelling using co-occurrence. This method yields friendship weight for each student. Friendship weight sorted into descending order to get close friend for predicting academic performance of each student.

This paper is organized as follows: Section 2 described each stages of our proposed method. In section 3, described the experimental result, evaluation and analysis. Finally, the conclusion is described in section 4 and provide the future work for the next research.

\section{METHOD}

Figure 1 shown the general framework of our proposed method in this system. There are four main steps in this system, that are calculate friendship weight, sorting into descending order, find k-best friend, and finally academic performance prediction process. Friendship weight calculated to get ratio communication frequency of each friend. Then, this weight sorted into descending order to get which friends closest to student. Then, best friend has highest weight than other students. Finally, GPA of closest friend and student is applied to academic performance prediction.

\section{A. Find Friendship Weights}

The main purpose of find friendship weight is get the ratio of communication frequency between students. We use comparison method because this method can show influence of each friends, which have various communication frequency. To generate this weight, the data input is co-occurrence matrices between students with $m x n$ size. Friendship weight can be determined by the following Equation. 1

$$
w(a, b)_{n}^{m}=\frac{\text { frequency }(a, b)}{\sum \text { frequency }(d t)}
$$

where $w(a, b)$ is friendship weight between students a and $\mathrm{b}, \operatorname{frequency}(a, b)$ is the number of communication frequency between students $\mathrm{a}$ and $\mathrm{b}$ and divide with $\sum$ frequency $(d t)$ which represent sum of communication frequency all students in the same location.

\section{B. Sorted into Descending Order}

Friendship weight that is obtained from previous steps is used to the second steps. Friendship weight is sorted into descending order. Ordered weight become a basic to find the closest friend. The highest weight showed the closer friendship. Otherwise, the smallest weight showed the furthest friend.

\section{Find k-Best Friend}

The ordered weight is applied in find k-best friend steps to obtain the k-closest friend of each students. We
TABLE 2.

EXPERIMENTAL RESULT FOR EFFECT OF THE NUMBER OF INCREASED DATA

\begin{tabular}{lll}
\hline \hline Location & Data & Accuracy (\%) \\
\hline Competition & 50 & 32 \\
& 100 & 43.43 \\
\hline \hline \multicolumn{2}{c}{ TABLE 3. } \\
& EXPERIMENT ON DIFFERENT MONTHS \\
\hline \hline Location & Month & Accuracy (\%) \\
\hline Competition & October & 21.25 \\
& November & 23.125 \\
& December & 21.25 \\
\hline \hline
\end{tabular}

must initialize the value of $\mathrm{k}$ parameters. In general, the number of best friends or $\mathrm{k}$ is not more than three persons. Then, the best friend GPA detail is given to the final steps. This GPA will be converted into academic predicate and will be the input for the final steps.

\section{Academic Performance Prediction}

Academic performance prediction is the final steps of this proposed method. The satisfactory result of academic performance prediction is obtained from best friend academic performance predicate. The GPA from all close friends will be averaged. Averaged GPA will be converted into academic performance predicate. Furthermore, for evaluation this result will be compared with each student academic performance predicate. This prediction will be evaluated using accuracy

\section{RESULTS AND DISCUSSION}

\section{A. Dataset Description}

Dataset that we used in this research is 160 student data with the number of interactions between student minimum one. This dataset used three months sample dataset before the end of semester. The dataset was modelled by co-occurrence theory. Co-occurrence shown that students raise a record at the same location [1]. Cooccurrence theory is illustrated in Figure 2.

Dataset that we used in our research have two symbols. As illustrated in Figure 3, \#1 means two students M0005 and M0000 co-occur one times in competition. Otherwise, \# means that two students M0029 and M0000 didn't occur in any competition. Thus in this research we collect these datasets in three-month i.e October, November, and December and used it to validate this research.

\section{B. Quantitative Evaluation}

This proposed method will be evaluated using accuracy. Accuracy is one of the simplest quantitative evaluation. Accuracy evaluated the result of proposed method (predicted value) that true predict and the real data or ground truth (actual value). The large value of accuracy means an effective performance. Accuracy can be determined by Equation. 2

$$
\text { Acc }=\frac{\text { sum of the true data }}{\text { sum all data }} \times 100 \%
$$

\section{Effect the Number of Best Friend}

The number of friends $k$ have been experimented to this proposed method with different values. As described earlier, this proposed method needs to determine the value of $k$ at the first stage. To determine the optimal 


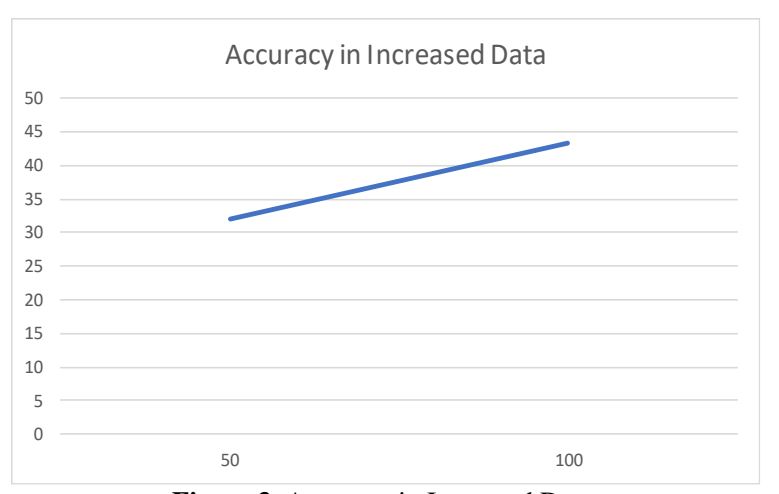

Figure 3. Accuracy in Increased Data.

TABLE 4 .

THE TOTAL COMPETITION

\begin{tabular}{lll}
\hline \hline Location & Month & Amount \\
\hline Competition & October & 5 \\
& November & 110 \\
& December & 0 \\
\hline
\end{tabular}

result, we experimented the academic performance prediction process with the number of $k 1,3,5,7$, on the sample data 50 students.

Table 1 shows the experimental results for effect of the number of friends. Can be seen in the table 1 that there are no changes in accuracy value. This condition can be affected by the dataset in experiment. Each student in dataset, doesn't have an interaction with other students more than one. So, when the number of $\mathrm{k}$ is increased, the average value of academic performance each student still same. We can present that each friend has same ratio to the student.

The best academic performance prediction depending on the number of interactions. The same number of interactions or communication frequency lead our proposed method can't detect the true proportion of friends influence to students.

\section{Effect the Number of Increased Data}

In this section, academic performance prediction using proposed method is experimented on different number of datasets. All the results were conducted on the number of $k=1$. Because in general, the number of best friends is not more than one student. The results will be verified using with the real academic performance dataset.

Table 2 shows the experimental results for effect of the number of increased datasets. Can be seen from table 2 that the accuracy values increased while dataset increased. This condition can be affected by increased dataset. So, the data is more varied.

\section{E. Experimented on Three Different Months}

In this section, academic performance prediction using our proposed method is experimented on three months. Experiments aim to validate friendships is a social dynamics relationship. Experiments were conducted on October, November, and December.

Table 3 shows the experimental results on three different months. Can be seen clearly in Figure $4 \mathrm{~s}$ that the accuracy value changed in every month. Even though there was a significant change in accuracy value, but the accuracy value was increased in November and decreased in December.

Table 4 shows the number of competitions that was held and followed by students. Can be seen clearly, that

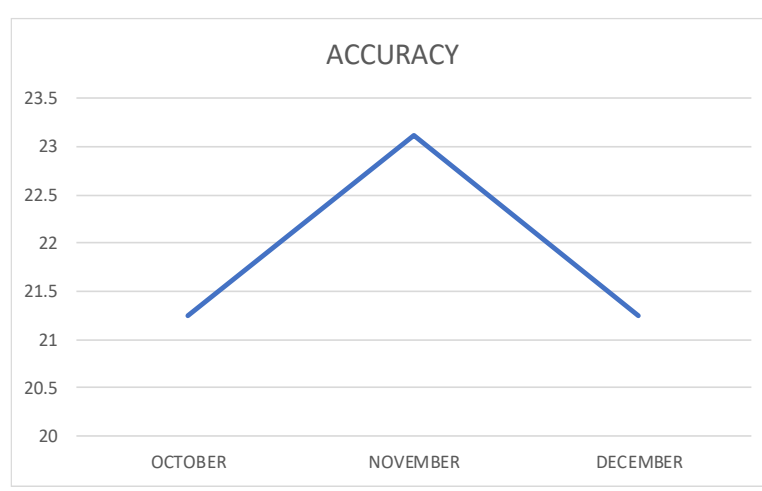

Figure 4. Experimental Result on Three Different Months.

the number of competitions that was held is different. The number of competitions in December tend to be decreased and November is the highest number of competitions. Decreased number of competitions is caused by this month is close to Final Exam. So, the student and the academic management was prepared to the Final Exam.

\section{F. Effect the Number of Best Friend}

The number of friends $k$ have been experimented to this proposed method with different values. As described earlier, this proposed method needs to determine the value of $k$ at the first stage. To determine the optimal result, we experimented the academic performance prediction process with the number of $k 1,3,5,7$, on the sample data 50 students.

Table 1 shows the experimental results for effect of the number of friends. Can be seen in the table 1 that there are no changes in accuracy value. This condition can be affected by the dataset in experiment. Each student in dataset, doesn't have an interaction with other students more than one. So, when the number of $\mathrm{k}$ is increased, the average value of academic performance each student still same. We can present that each friend Academic performance prediction is the final steps of this proposed method. The satisfactory result of academic performance prediction is obtained from best friend academic performance predicate. The GPA from all close friends will be averaged. Averaged GPA will be converted into academic performance predicate. Furthermore, for evaluation this result will be compared with each student academic performance predicate. This prediction will be evaluated using accuracy

\section{CONCLUSION}

This paper presented and evaluated a proposed method for predicting academic performance based on friend relationship weighting from communication frequency at following competition. Initially, the dataset is preprocessed by researcher into co-occurrence matrices to get representative data and easy to calculate interaction of each student. The co-occurrences matrices are processed to find the friendship weight of each students. After that, friendship weight sorted into descending order to find the closest friend of each students. Then, closest friend GPA is used to predict student academic performance.

Proposed method, can successfully predicting student academic performance with different number of close friends, increasing dataset, and validate friendship is dynamics social relationship. The number of 


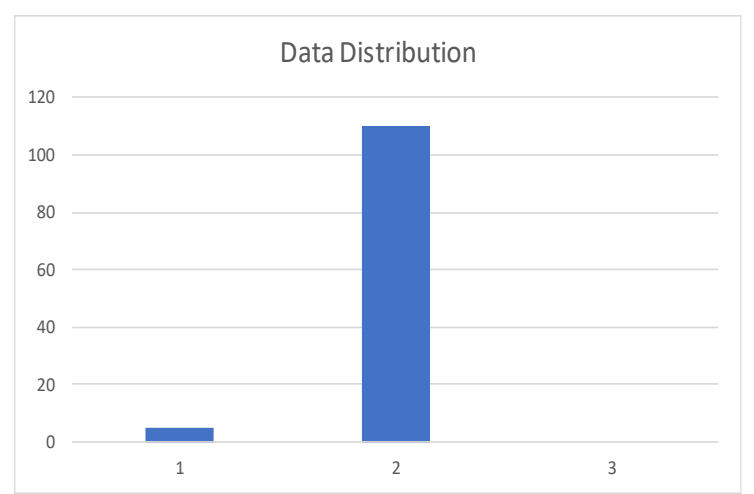

Figure 5. Data Distribution of Competition.

communication frequency is the important feature in this proposed method. Because, this feature sees proportion of friend influence. So, the successfully of this propose method depending on the number of interaction or communication frequency each student. Second experiment is done by increasing dataset. From the experimental result, the accuracy value increase while the number of dataset increase. The proposed method successfully predicts student academic performance while data more varied.

Lastly, the proposed method evaluated in three different months. The proposed method successfully predicts academic performance in different month. From the experimental result, the biggest value of accuracy is in November. As described before, these phenomena can be caused by the number of competitions held in November. November is the most effective day for activities outside academic activities. So, the interaction in November is high. For further research, we aim to implemented on more meeting location and considering duration communication to get more representative friend relationship weighting.

\section{ACKNOWLEDGEMENT}

We are very thankful to Institut Teknologi Sepuluh Nopember (ITS) for the opportunity to do this research and proposed a method for friend relationship weight.

\section{REFERENCES}

H. Yao and et al, "Database Systems for Advanced Applications," pp. 597-609, 2017.

[2] W. R., G. Harari, P. Hao, X. ZZhou, and A. . Campbell, "SmartGPA: how smartphones can assess and predict academic performance of college students," in Proceedings of the 2015 ACM International Joint Conference on Pervasive and Ubiquitous Computing (UbiComp), 2015.

[3] J. Flashman, "Academic achievement and its impact on friend dynamics," Sociol. Educ., vol. 85, no. 1, pp. 61-80, 2012.

[4] G. Mesch and I. Talmud, "The quality of online and offline relationships: The role of multiplexity and duration of social relationships," Inf. Soc., vol. 22, no. 3, pp. 137-148, 2006

[5] R. Cheng, J. Pang, and Y. Zhang, "Inferring Friendship from Check-in Data of Location-Based Social Networks," in Proceedings of the 2015 IEEE/ACM International Conference on Advances in Social Network Analysis and Mining 2015 - ASONAM '15, 2015, pp. 1284-1291. 